\begin{tabular}{|c|c|c|}
\hline & $\begin{array}{c}\text { European Association for the } \\
\text { Development of Renewable Energies, Environment and } \\
\text { Power Quality (EA4EPQ) }\end{array}$ & $\begin{array}{l}\text { International Conference on Renewable Energies and Power Quality } \\
\text { (ICREPQ'12) } \\
\text { Santiago de Compostela (Spain), 28th to 30th March, } 2012\end{array}$ \\
\hline
\end{tabular}

\title{
A Novel Power Quality Monitor Placement Method Using Adaptive Quantum- Inspired Binary Particle Swarm Optimization
}

\author{
A. A. Ibrahim, Student Member, IEEE, A. Mohamed, Senior Member, IEEE, H. Shareef
}

Department of Electrical, Electronic and Systems Engineering

University Kebangsaan Malaysia

Bangi, Selangor 432600, Malaysia

Phone/Fax number:+60389216006/60389202314, e-mail: asrul@eng.ukm.my;azah@eng.ukm.my;shareef@eng.ukm.my

\begin{abstract}
This paper presents a novel method for solving optimal power quality monitor placement problem in monitoring voltage sags in power systems using the adaptive quantum-inspired particle swarm optimization (PSO). The optimization considers multi objective functions and handles observability constraint determined by the concept of the topological monitor reach area. The overall objective function consists of two functions which are based on monitor overlapping index and sag severity index. In this algorithm, the standard quantum-inspired binary PSO is modified by applying the concept of artificial immune system as an adaptive element to make it more flexible towards better quality of solution and computational speed. The proposed algorithm is applied on the IEEE 30-bus transmission system and the IEEE 34-node distribution system and compared to the conventional PSO.
\end{abstract}

Index Terms. Adaptive quantum-inspired PSO, voltage sag, topological monitor reach area, artificial immune system.

\section{Introduction}

Power quality is a prominent issue which demands utilities to deliver good quality of electrical power to end users. Among all power disturbances, voltage sags are the most frequently occurring disturbance which give severe impact on sensitive loads. Voltage sags are usually monitored by means of the conventional power quality monitoring practice in which power quality monitors (PQMs) are installed at all buses in a power distribution network. The disadvantage of this approach is the widespread installation of PQMs. Reducing the number of monitors will reduce the total cost of power quality monitoring system and also reduces redundancy of data being measured by monitors [1]. Thus, methods are required for determining minimum number and the strategic location of PQMs to ensure that voltage sags are captured by the monitors. In [2]-[5], the concept of monitor observability is utilized to find optimal placement of PQMs in transmission systems. However, this concept is not suitable for radial distribution networks. Therefore, there is a need to develop a new optimal PQM placement method that is applicable for both transmission and distribution systems.
A few optimization techniques have been used to solve the optimal PQM placement problem in the last few years. In [2], the PQM placement method was developed by using the GAMS software as an integer linear program. In [3], the branch and bound algorithm is applied by dividing the solution space into smaller spaces to make it easier to solve. However, it may give totally a wrong solution when there is a mistake in selecting a branch in earlier stages. In [4], genetic algorithm (GA) is used for solving the optimal PQM placement problem. However, the disadvantage of GA is that it is slow in terms of convergence rate. Thus, an alternative optimization technique with faster convergence rate such as particle swarm optimization (PSO) [5] is suggested to be implemented. The main aim of this study is to develop a new algorithm for solving the optimal PQM placement problem in power systems by considering three concepts, namely, quantum behavior, binary PSO and artificial immune system. In the proposed algorithm, the observability concept is introduced which is mainly based on the topological monitor reach area (TMRA) which makes observability applicable for both transmission and distribution systems. Besides that, the monitor coverage control parameter, $\alpha$, is used to give greater flexibility to the search algorithms in complying with sensitivity and economic capability. The parameter $\alpha$ is defined as a voltage threshold level in p.u. at a monitored bus to indicate whether a fault occurs inside or outside the monitor's coverage area. A PQM usually detects and captures voltage variations when the measured RMS voltage reaches 0.9 p.u.. In this study, the maximum $\alpha$ value is suggested to be set at 0.85 p.u. so as to allow some overlapping of the monitor coverage area at the boundary. This approach will help to overcome the boundary issues and non-monitored fault on the line segment at the boundary.

\section{The Monitor Coverage Concept}

The monitor coverage is the most important entity in the determination of PQM placement as it is used to evaluate the placement so as to guarantee the observability of the whole 
power network. The monitoring coverage concept is called the monitor reach area (MRA) [2]. In the formation of the MRA, residual voltages at each bus for all fault cases are required. The residual voltages are saved in a data storage called as the Fault Voltage (FV) matrix where the matrix columns $(j)$ represent the bus numbers of residual voltages and the matrix rows $(k)$ relate to the simulated fault of specific type and position that causes voltage sags [3]. Then, the MRA matrix can be obtained by comparing all the FV matrix elements for each phase with the threshold value, $\alpha$. Each element of the MRA matrix is filled with 1 (one), when the voltage goes below or equal to $\alpha$ p.u. in any phase and with 0 (zero) otherwise as given by,

$$
\operatorname{MRA}(j, k)=\left\{\begin{array}{l}
1, \text { if } \operatorname{FV}(j, k) \leq \alpha \text { p.u. at any phase } \\
0, \text { if } \operatorname{FV}(j, k)>\alpha \text { p.u. at all phases }
\end{array} \forall j, k\right.
$$

In this study, a topological monitor reach area (TMRA) is introduced to make it applicable for both distribution and transmission systems. The TMRA matrix is a combination of MRA matrix and topology (T) matrix by using operator 'AND' and is expressed as follows:

$$
\operatorname{TMRA}(j, k)=\operatorname{MRA}(j, k) \bullet \mathrm{T}(j, k)
$$

Similar to MRA and FV matrices, the $\mathrm{T}$ matrix columns represent bus number and its rows are correlated to fault location and type of fault. The T matrix is constructed based on the concept of paths in graph theory. During a fault, the faulted bus voltage level will fall to nearly ground level and becomes a cut vertex. At this moment, the faulted bus can be separated into several independent vertices corresponding to the number of branches connected to the bus. Thus, a path will be considered when at least one route from start vertex to end vertex which does not go through the cut vertex is available. In this case, each generating station can be a start vertex and a bus under consideration for PQM placement can be an end vertex. According to the condition, $\mathrm{T}$ matrix is filled with 1 (one) when there is a path from any generating bus to a particular bus under consideration and 0 (zero) otherwise.

\section{Optimal PQM Placement Formulation}

There are three common elements required in the binary optimization technique, namely, decision vectors, objective function and optimization constraints. Thus, each element is formulated and explained in order to obtain the optimal solution for the PQM placement. The optimization explores the optimal solution as defined in the objective function through the bits manipulation of decision vector subject to the optimization constraints in each generation. The process is iterated for a fixed number of times or until a convergence criterion is achieved.

\section{A. Decision Vector}

To satisfy the solution process in this study, the Monitor
Placement (MP) vector is introduced to represent the binary decision vector $\left(x_{i j}\right)$ in bits in the optimization process. The bits of this vector indicate positions of monitors that are needed or not in power system network. The dimension of the vector corresponds to the number of buses in the system. A value 0 (zero) in the MP (n) indicates that no monitor is needed to be installed at bus $n$ whereas a value 1 (one) indicates that a monitor should be installed at bus $n$. Thus, the MP vector is described by the following expression;

$$
\operatorname{MP}(n)=\left\{\begin{array}{l}
1, \text { if PQM is required at bus } n \\
0, \text { if PQM is not required at bus } n
\end{array} \forall n\right.
$$

\section{B. Objective Function}

The objective function is formulated to solve two objectives, namely, optimal number of required monitors and optimal locations to install the monitors. The number of required monitors (NRM) to be minimized can easily be obtained and expressed as,

$$
\mathrm{NRM}=\sum_{n=1}^{N} \mathrm{MP}(n)
$$

To determine the best placement to install the monitors, additional parameters are required to achieve the goals. The placement of PQMs in a power system will result in different overlaps of monitor coverage areas for different arrangements. Here, it is important to note that these overlaps indicate the number of monitors which record the same fault occurrence in a power system. Therefore, these overlaps should be minimized. The overlaps can be calculated by multiplying the TMRA matrix and the transposed MP vector. If all the elements in the obtained results are 1, it implies that there is no overlap in the monitors' coverage. Thus, monitor overlapping index (MOI) is introduced to evaluate the best monitor arrangement in a power system. A lower MOI value indicates a better arrangement of PQMs in a power system. The MOI is given by,

$$
\mathrm{MOI}=\frac{\sum\left(\mathrm{TMRA}^{*} \mathrm{MP}^{\mathrm{T}}\right)}{\mathrm{NFLT}}
$$

where NFLT is the total number of fault locations considering all types of faults.

However, the MOI alone is not enough to provide a good solution in determining the best placement of monitors. As a result, another index which is called the Sag Severity Index (SSI) is considered. This index defines the severity level of a specific bus towards voltage sag, where any fault occurrence causes a big drop in voltage magnitudes for most of the buses in the system. Therefore, the severity level (SL) should be determined first and it is given by,

$$
\mathrm{SL}^{(\mathrm{t})}=\frac{\mathrm{N}_{\mathrm{SPB}}}{\mathrm{N}_{\mathrm{TPB}}}
$$


where,

$\mathrm{N}_{\mathrm{SPB}}$ : Number of phases experiencing voltage sag with magnitudes below t p.u.;

$\mathrm{N}_{\mathrm{TPB}}$ : Number of phases in total for the system.

Then, the SSI is obtained by applying weighting factors for different SLs. The SL with the lowest threshold $t$ value is assigned with the highest weighting factor and vice versa. In this case, five thresholds are considered as $0.1,0.3,0.5,0.7$ and 0.9 p.u. Then, SSI can be calculated as expressed in (7) where the number 5 refers to weighting factor levels and the value 15 corresponds to the total weight. Finally, the calculated SSI value is stored in a matrix form where the matrix columns correlate to the bus number and the matrix rows correlates to the type of fault $(\mathrm{F})$. A higher value of SSI indicates a better placement of monitor.

$$
\mathrm{SSI}^{\mathrm{F}}=\frac{1}{15} \sum_{k=1}^{5} k * \mathrm{SL}^{\left(1-\frac{2 k-1}{10}\right)}
$$

To combine the MOI and SSI indices, both of them should have similar optimal criteria of either maximum or minimum. In this case, the SSI matrix is modified to give a minimum criterion in optimization to make it similar to the case of minimization of MOI. It is important to note that a maximum value of SSI element is equal to 1 . Thus, it can be obtained by using complementary matrix of SSI. Then, a negative severity sag index (NSSI) is introduced to evaluate the best placement of monitors in the system. The NSSI can be obtained using (8). As a result, a lower NSSI value indicates a better arrangement of PQMs in the system.

where,

$$
\mathrm{NSSI}=\frac{\sum\left\lfloor(\mathrm{ONE}-\mathrm{SSI}) * \mathrm{MP}^{\mathrm{T}}\right\rfloor}{\mathrm{NFT}}
$$

ONE: Matrix with all entries ' 1 ' where its dimension is the same as the SSI matrix;

NFT: Number of fault types.

All the above functions can be combined in a single objective function by using the summation method since all the functions have similar optimal criteria. However, the objective functions should be independent and should not influence each other in finding the optimal solution. The single multiobjective function to solve optimization problems in this study is expressed in (9). In this equation, multiplication between NRM and MOI will never go below the value of NSSI. Inherently, the MOI is given higher priority in determining optimal monitor placement as compared to NSSI value. The concept is based on weighted sum method that has been commonly used to solve multi-objective problems [6].

$$
f=(\mathrm{NRM} \times \mathrm{MOI})+\mathrm{NSSI}
$$

\section{Optimization Constraints}

The optimization algorithm must run while satisfying all the constraints that are used to find optimal number of PQMs for the system. As given in (10), the multiplication of the TMRA matrix by the transposed MP matrix gives the number of monitors that can detect voltage sags due to a fault at a specific bus. If one of the resulting matrix elements is 0 (zero) then it means that no monitor is capable of detecting sag caused by faults at a particular bus, whereas if the value is greater than 1 (one), that means more than one monitor have observed a fault at the same bus. For that reason, the following restrictions must be fulfilled to make sure that each fault is observed by at least one monitor;

$$
\sum_{\mathrm{i}=1}^{k} \operatorname{TMRA}(k, i) * \mathrm{MP}(i) \geq 1 \quad \forall k
$$

\section{Adaptive QBPSO Algorithm}

Evolutionary computation techniques are evolving rapidly in solving optimization problems because they are found to be more robust and efficient in optimizing multidimensional problems in various fields [5]. In this study, a novel optimization algorithm called as the adaptive quantuminspired binary particle swarm optimization (AQBPSO) which is an improvement from the existing QBPSO algorithm is introduced.

\section{A. Binary Particle Swarm Optimization}

A binary particle swarm optimization (BPSO) was originally developed and introduced by Kennedy and Eberhart [7]. The original conventional PSO was designed to solve in continuous valued space. It is a random search algorithm that simulates natural evolutionary process by mimicking the social behaviour of birds, bees or a school of fishes. In BPSO, a j-th bit of the $\mathrm{i}$-th particle $\left(x_{i j}\right)$ in the swarm is represented as a bit 0 or 1 in MP vector whereas its movement in the space is in real value which is known as velocity vector $\left(v_{i j}\right)$. The PSO operators update the particle velocity's bits based on current velocity, the best position explored so far (P) and the global best position explored by swarm (G) as given in (11). Then, a new particle's position $\left(x_{i j}\right)$ is updated using a sigmoid function [7].

$v_{i j}(t+1)=w \cdot v_{i j}(t)+c_{1} \varphi_{1}\left(P_{j}-x_{i j}(t)\right)+c_{2} \varphi_{2}\left(G_{j}-x_{i j}(t)\right)$

where,

$w$ : inertia weight which decreases monotonously

from $w_{\max }$ to $w_{\min }$ along iteration;

$c_{1}, c_{2}$ : positive acceleration coefficients;

$\varphi_{1}, \varphi_{2}$ : uniform random variables in interval $[0,1]$. 


\section{B. Quantum-Inspired Computing}

The first quantum inspired computing method was introduced by Moore and Nayaranan [8]. It is a numerical computational method by utilizing the principle of quantum mechanics. The smallest unit for quantum computing which is known as quantum bit (Q-bit) may be in the " 1 " state, in the " 0 " state or in superposition of the two corresponding to weighting factors of complex number $(\alpha, \beta)$ as represented in (12). The $|\alpha|^{2}$ and $|\beta|^{2}$ in the representation give a probability that the Q-bit will be in the " 0 " state and the " 1 " state, respectively [9]. Thus, the state can be normalized to unity as $|\alpha|^{2}+|\beta|^{2}=1$.

$$
|\Psi\rangle=\alpha|0\rangle+\beta|1\rangle
$$

Similar to particle's position in BPSO, all decision variables $\left(x_{i j}\right)$ can be represented by a string of Q-bits as single representation called Q-bit individual. In the quantum computing, the Q-bit individual is updated using a quantum gate (Q-gate) which is basically a unitary operator. $U$ is the rotation gate, the NOT gate, the controlled NOT gate or the Hadamard gate etc. used to change the probability of the Q-bit state so as to promise a reversible of the formation.

\section{Quantum-Inspired BPSO}

A Quantum-Inspired Binary Particle Swarm Optimization (QBPSO) is one of the most recent heuristic optimization that applies quantum mechanic behavior in the BPSO algorithm [10]. In the QBPSO, the rotation angle $(\Delta \theta)$ is utilized to replace the velocity updating procedure in the BPSO, a magnitude of the rotation angle $(\theta)$ is used to replace the inertia weight and the two acceleration coefficients and two random variables are replaced by two decision parameters, $\gamma_{1}$ and $\gamma_{2}$. The QBPSO operators update particle position bit $\left(x_{i j}\right)$ by using probability of $|\beta|^{2}$ stored in the Q-bit individual string which has already been updated by the rotation gate as shown in (16). The following are equations used in the QBPSO to search for the optimal solution:

$$
\Delta \theta_{i j}(t+1)=\theta \times\left[\gamma_{1}\left(P_{j}-x_{i j}(t)\right)+\gamma_{2}\left(G_{j}-x_{i j}(t)\right)\right]
$$

where,

$$
\begin{aligned}
& \gamma_{1}=\left\{\begin{array}{l}
1, \text { if } f(P) \leq f\left(x_{i}\right) \\
0, \text { otherwise; }
\end{array}\right. \\
& \gamma_{2}=\left\{\begin{array}{l}
1, \text { if } f(G) \leq f\left(x_{i}\right) \\
0, \text { otherwise }
\end{array}\right.
\end{aligned}
$$

$\theta$ : magnitude of rotation angle which monotonously decreases from $\theta_{\max }$ to $\theta_{\min }$ along iteration.

$$
\left[\begin{array}{c}
\alpha_{i j}(t+1) \\
\beta i j(t+1)
\end{array}\right]=U\left(\Delta \theta_{i j}(t+1)\right) \times\left[\begin{array}{c}
\alpha_{i j}(t) \\
\beta_{i j}(t)
\end{array}\right]
$$

$$
x_{i j}(t+1)=\left\{\begin{array}{l}
1, \text { if } r<\left|\beta_{i j}(t+1)\right|^{2} \\
0, \text { otherwise }
\end{array}\right.
$$

where,

$$
r: \text { an uniform random variable in interval }[0,1] \text {. }
$$

\section{D.Adaptive Quantum-Inspired BPSO}

In the optimizations procedure, there is a rejection process which takes place in the algorithm when the suggested solution does not fulfill the optimization constraints and it is required to search for another feasible solution. It may take a long time for searching the solution when possibility to get feasible combinations is very limited. In this study, increase of the parameter $\alpha$ will increase the sensitivity of the monitoring scheme and the available number of feasible solutions will be reduced. Thus, it needs adaptive process to make the algorithm more flexible and maintain the computational time. The idea of adaptive process is mainly based on immune system response of the T-cells against foreign pathogens in the artificial immune system (AIS) concept [11]. The immune system is responsible to neutralize all pathogenic effects or to destroy the infected cells which are classified as 'non-self' cells. The neutralization mechanism seems more suitable to be applied to the problem in this study. In this mechanism, the infected cell produces non self-antigens and self-antigens and then displays the antigens on its surface. At the same time, the T-cells with specific receptor are stochastically produced in Thymus (immune organ). Only T-cells in which their receptor matches with non-self antigens are released to the blood stream after going through negative selection process in the Thymus. Then, each T-cell binds with recognized non-self antigens and becomes inactivated antigen (non-toxic). These T-cells could not bind with self antigens since their receptor unmatched. Finally, the infected cell is totally neutralized when all non-self antigens on the infected cell's surface are bonded and it will be recognized as a 'self' cell..

In this problem, the MP vectors which are produced in the optimization algorithm will be recognized as non-self cells when they do not fulfill the constraints in (12) and self cells, otherwise. Instead of changing the whole string of the MP vector, bits manipulating can be done to adapt with the constraints. The manipulation can be done by randomly replacing one (1) in the non-self MP vector which represents binding mechanism between T-cells and non-self antigens. Besides that, the replacement is specified to locations of entries ' 1 ' in the particular row of TMRA which does not fulfill the constraints. The determination is based on the condition where the constraint is not fulfilled only if there is a mismatch between entries ' 1 ' in the particular TMRA row and MP vector. This determination process follows the negative selection in Thymus to produce specific T-cells to attack the target antigens. That means the particular constraint will be fulfilled after entry ' 1 ' is placed at one of the locations. The process will stop when the MP vector is fully adapted to the 
optimization constraints.

\section{Results and Discussion}

The adaptive QBPSO which is called AQBPSO, is then implemented and compared with the conventional QBPSO so as to illustrate its effectiveness with respect to different $\alpha$ values. Two test systems are used in this study, namely, the IEEE 30-bus transmission system and the IEEE 34-node distribution system. Three-phase (LLL) faults, double-line to ground (DLG) faults and single-phase to ground (SLG) faults were simulated at each bus in the systems with $0 \Omega$ fault impedance using the DIgSILENT software.

\section{A. Case I: IEEE 30-bus System}

The IEEE 30-bus test system is a balanced transmission system with 60 lines at two voltage levels; $132 \mathrm{kV}$ and $33 \mathrm{kV}$. There are 2 generating stations, 3 sychronous condensers and 4 step-down transformers. The IEEE 30-bus test system data are provided in [12]. Table I shows optimal number of PQM in the IEEE 30-bus system at different $\alpha$ values and the required computational times by QBPSO and AQBPSO to obtain the optimal solutions. From this table, both of these techniques provide the same optimal number of PQMs but with different computation times. It shows that AQBPSO requires longer time as compared to QBPSO when $\alpha$ is set at 0.85 p.u. and becomes comparable when $\alpha$ value is set to 0.75 p.u.. This may be due to many alternative solutions available in the solution space which are not critical enough to randomize the process in QBPSO to provide a feasible PQM placement. Besides that, AQBPSO needs to check for each row of the TMRA matrix in order to provide a specific and particular correction process in fulfilling the optimiztion constraints. After decreasing the $\alpha$ value below 0.65 p.u., the AQBPSO gives optimal PQM placement results in just a few seconds even after tuning $\alpha$ to a very lower values. Table II shows the optimal PQM placement results of AQBPSO and QBPSO in terms of the bus locations to install the PQMs. The results showed that the bus locations for placing the PQMs are similar for both AQBPSO and QBPSO.

Table I. - Performance of QBPSO And AQBPSO on The 30-Bus System At Different $\alpha$ Values

\begin{tabular}{ccccc}
\hline \hline \multirow{2}{*}{$\begin{array}{c}\text { value } \\
\text { value } \\
\text { (p.u.) }\end{array}$} & $\begin{array}{c}\text { Number } \\
\text { of PQMs }\end{array}$ & $\begin{array}{c}\text { Elapsed } \\
\text { Time (s) }\end{array}$ & $\begin{array}{c}\text { Number } \\
\text { of PQMs }\end{array}$ & $\begin{array}{c}\text { Elapsed } \\
\text { Time } \\
(\mathrm{s})\end{array}$ \\
\hline 0.85 & 1 & 0.75 & 1 & 1.805 \\
0.75 & 3 & 1.63 & 3 & 1.940 \\
0.65 & 6 & 3.31 & 6 & 2.154 \\
0.55 & 8 & 21.23 & 8 & 3.680 \\
0.45 & 11 & 137.53 & 11 & 4.093 \\
0.35 & 17 & 4668.46 & 17 & 4.286 \\
0.25 & 19 & 55084.46 & 19 & 4.395 \\
\hline \hline
\end{tabular}

Table II- Optimal placement results of QBPSO and AQBPSO on the 30-bus System in terms of bus locations

\begin{tabular}{ccc}
\hline \hline \multirow{2}{*}{$\begin{array}{c}\alpha \text { value } \\
\text { (p.u.) }\end{array}$} & \multicolumn{2}{c}{ PQM Placement (bus) } \\
\cline { 2 - 3 } 0.85 & 25 & AQBPSO \\
0.75 & $5,20,25$ & 25 \\
0.65 & $1,5,11,15,25,29$ & $1,5,11,15,25,29$ \\
0.55 & $4,7,11,15,17$, & $4,7,11,15,17,20$, \\
& $20,26,29$ & 26,29 \\
0.45 & $2,4,7,11,13,14$, & $2,4,7,11,13,14$, \\
& $16,19,23,26,29$ & $16,19,23,26,29$ \\
\hline \hline
\end{tabular}

Table III shows the performance comparison between the AQBPSO and the QBPSO in terms of quality of solution, convergence rate and computational time after performing 20 runs at $\alpha=0.45$ p.u. for the transmission system. As can be seen in the table, overall the AQBPSO gives better performance than QBPSO as indicated by the average values. Based on the standard deviation $(\sigma)$, both of the algorithms provide a precise solution. In terms of computational times, AQBPSO is much faster than QBPSO. The result has also illustrated that AQBPSO converge faster than QBPSO in which it has solved the optimization problem in 4 iterations compared to the QBPSO in 7 iterations.

Table III - Performance of QBPSO and AQBPSO on the 30-bus System for $\alpha$ at 0.45 p.u.

\begin{tabular}{clcccc}
\hline \hline \multicolumn{2}{c}{ Item } & Worst & Average & Best & $\sigma$ \\
\hline \multirow{3}{*}{ QBPSO } & Fitness & 28.67 & 28.41 & 28.27 & 0.187 \\
& Iteration & 91 & 25.25 & 7 & 24.22 \\
& Time (s) & 185.16 & 91.90 & 40.11 & 32.78 \\
\hline \multirow{3}{*}{ AQBPSO } & Fitness & 28.67 & 28.37 & 28.27 & 0.168 \\
& Iteration & 78 & 16.95 & 4 & 15.47 \\
& Time (s) & 4.18 & 4.12 & 4.05 & 0.026 \\
& & & & & \\
\hline
\end{tabular}

\section{B. Case II: IEEE 34-node System}

The IEEE 34-node test system is an unbalanced distribution system. The system consists of 34 nodes interconected by 34 lines and the test system data is provided in [13]. Table IV shows the optimal number of PQMs in the IEEE 34-node system at different $\alpha$ values and the computational times by QBPSO and AQBPSO to obtain the optimal solutions. As can be seen in this table, in terms of computational time, the AQBPSO is faster than the QBPSO as the $\alpha$ value decreases. For the $\alpha$ value greater than 0.55 p.u., the compuational times by QBPSO and AQBPSO are comparable. Table VI shows the optimal PQM placement results of AQBPSO and QBPSO to indicate which buses the PQMs should be installed. The results showed that the bus locations for placing the PQMs in the 34 
node system are similar for both AQBPSO and QBPSO.

Table IV- Performance of QBPSO and AQBPSO on 34-node System at Different $\alpha$ Value

\begin{tabular}{ccccc}
\hline \hline \multirow{2}{*}{$\begin{array}{c}\alpha \\
\text { value } \\
\text { (p.u.) }\end{array}$} & $\begin{array}{c}\text { Number } \\
\text { of PQMs }\end{array}$ & $\begin{array}{c}\text { Elapsed } \\
\text { Time (s) }\end{array}$ & $\begin{array}{c}\text { Number } \\
\text { of PQMs }\end{array}$ & $\begin{array}{c}\text { Elapsed } \\
\text { Time } \\
(\mathrm{s})\end{array}$ \\
\hline 0.85 & 3 & 1.15 & 3 & 2.723 \\
0.75 & 3 & 1.39 & 3 & 2.782 \\
0.65 & 4 & 1.63 & 4 & 2.762 \\
0.55 & 5 & 3.09 & 5 & 3.265 \\
0.45 & 6 & 4.38 & 6 & 3.513 \\
0.35 & 8 & 17.13 & 8 & 3.786 \\
0.25 & 9 & 87.47 & 9 & 3.878 \\
0.15 & 13 & 710.25 & 13 & 4.026 \\
0.05 & 18 & 34632.31 & 18 & 4.189 \\
\hline \hline
\end{tabular}

Table VI shows the performances of the algorithms in terms of convergence rate and computational time after performing 20 runs at $\alpha=0.25 \mathrm{p}$.u. for the distribution system. In this case, AQBPSO requires more iterations to converge than QBPSO but in terms of computational time it is faster. In terms of the obtained solution, both the AQBPSO and the QBPSO give the same the best fitness values.

Table V - Optimal placement results of QBPSO and AQBPSO on the 34-node System in terms of bus locations

\begin{tabular}{ccc}
\hline \hline \multirow{2}{*}{$\begin{array}{c}\alpha \text { value } \\
\text { (p.u.) }\end{array}$} & \multicolumn{2}{c}{ PQM Placement (bus) } \\
\cline { 2 - 3 } 0.85 & $800,808,832$ & AQBPSO \\
0.75 & $800,812,846$ & $800,808,832$ \\
0.65 & $800,808,814,888$ & $800,808,814,888$ \\
& $800,808,814,852$, & $800,808,814,852$, \\
0.55 & 890 & 890 \\
& $800,808,812,832$, & $800,808,812,832$, \\
0.45 & 850,890 & 850,890 \\
& $800,808,812,822$, & $800,808,812,822$, \\
0.35 & $848,850,854,890$ & $848,850,854,890$ \\
\hline \hline
\end{tabular}

Table VI - Performance of QBPSO and AQBPSO on 34-node System for $\alpha$ at 0.25 p.u.

\begin{tabular}{clcccc}
\hline \hline \multicolumn{2}{c}{ Item } & Worst & Average & Best & $\sigma$ \\
\hline \multirow{4}{*}{ QBPSO } & Fitness & 26.10 & 23.16 & 23.01 & 0.692 \\
& Iteration & 30 & 13.15 & 8 & 5.669 \\
& Time $(\mathrm{s})$ & 182.03 & 111.98 & 76.35 & 34.59 \\
\hline \multirow{2}{*}{ AQBPSO } & Fitness & 23.01 & 23.01 & 23.01 & 0.000 \\
& Iteration & 72 & 15.85 & 7 & 13.89
\end{tabular}

$\begin{array}{lllll}\text { Time (s) } & 3.991 & 3.956 & 3.896 & 0.021\end{array}$

\section{Conclusion}

This paper presented a comparative performance of AQBPSO and QBPSO in solving the multi-objective optimization for optimal PQM placement in distribution and transmission test systems. The optimization problem formulation is mainly based on the use of the TMRA and the two placement evaluation indices, namely, the SSI and the MOI. The optimization techniques have been tested on the IEEE 30-bus and IEEE 34-node test systems for determining the best optimal PQM placements at different voltage threshold levels, $\alpha$. The comparative results reveal that the AQBPSO gives better optimal PQM placement in terms of computational speed and maintain good quality of solution.

\section{References}

[1] M.A. Eldery, E.F. El-Saadany and M.M.A. Salama, "Optimum Number and Location of Power Quality Monitors," in Proc. 2004 11th Int. Conf. on Harmonics and Quality of Power, pp. 50-57.

[2] G. Olguin, F.Vuinovich, and M.H.J. Bollen, "An Optimal Monitoring Program for Obtaining Voltages Sag System Indexes," IEEE Trans. Power Syst., vol. 21, n. 1, pp. 378-384, Feb. 2006.

[3] D.C.S. Reis, P.R.C. Villela, C.A. Duque and P.F. Ribeiro, "Transmission Systems Power Quality Monitors Allocation," in IEEE 2008 Power and Energy Society General Meeting Conversion and Delivery of Electrical Energy in the $21^{\text {st }}$ Century, pp. 1-7.

[4] C.F.M. Almedia and N. Kagan, Allocation of Power Quality Monitors by Genetic Algorithms and Fuzzy Sets Theory, in Proc. 2009 15th Int. Conf. on Intelligent System Applications to Power Systems, pp. 1-6.

[5] E. Elbeltagi, T. Hegazy and D. Grierson, "Comparison Among Five Evolutionary-Based Optimization Algorithms," Advanced Engineering Informatics, vol. 19, n. 1, pp. 43-53, Jan. 2005.

[6] R.T. Marler and J.S. Arora, "The Weighted Sum Method for Multi-Objective Optimization: New Insights," Structural and Multidisciplinary Optimization, vol. 41, n. 6, pp. 853-862, June 2010.

[7] J. Kennedy and R.C. Eberhart, "A Discrete Binary Version of The Particle Swarm Algorithm," in Proc. 1997 IEEE Int. Conf. on Systems, Man and Cybernics, vol. 5, pp. 4104-4108.

[8] M. Moore and A. Nayaranan, Quantum-Inspired Computing, Dept. Computer Science, University Exeter. Exeter, U.K., 1995.

[9] T. Hey, "Quantum computing: An introduction," in Computing and Control Engineering Journal, Piscataway, NJ: IEEE Press, 1999, vol. 10, n. 3, pp. 105-112.

[10] Y.-W. Jeong, J.-B. Park, S.-H. Jang and K.Y. Lee, "A New Quantum-Inspired Binary PSO: Application to Unit Commitment Problems for Power Systems," IEEE Trans. Power Syst., vol. 25, n. 3, pp. 1486-1495, Aug. 2010.

[11] U. Aickelin and D. Dasgupta, "Artificial Immune System," in Search Methodologies: Introductory Tutorials in Optimization and Decision Support Techniques, E.K. Burke and G. Kendall, Ed. New York: Springer, 2005, pp. 375-399.

[12] R. Christie, "The IEEE 30 Bus Test Case," Internet: www.ee.washington.edu/research/pstca/pf30/pg_tca30bus.htm, Aug. 1993 [May 13, 2010]. 
[13] B. Kersting, R. Durgan and S. Carneioro, Jr., "Distribution Test Feeders: 34-bus Feeder," Internet:

http://ewh.ieee.org/soc/pes/dsacom/ testfeeders/index.html. [Apr. 20, 2010]. 